\title{
Xantonas, triterpenos e atividade antibacteriana do extrato em diclorometano de Kielmeyera cuspidata Saddi, Clusiaceae
}

\author{
Ivoneide S. Sobral, ${ }^{1}$ Lourdes C. Souza-Neta, ${ }^{1}$ Giselle. A. N. Costa, ${ }^{1}$ \\ Maria L. S. Guedes, ${ }^{2}$ Dirceu Martins, ${ }^{1}$ Frederico G. Cruz ${ }^{* 1}$ \\ ${ }^{1}$ Grupo de Estudos de Substâncias Naturais Orgânicas, Instituto de Química, \\ Universidade Federal da Bahia, 40170-290 Salvador-BA, Brasil \\ ${ }_{2}^{2}$ Instituto de Biologia, Universidade Federal da Bahia, Campus de Ondina, 40170-290 Salvador-BA, Brasil
}

\begin{abstract}
RESUMO: Do extrato em diclorometano do tronco de Kielmeyera cuspidata Saddi, Clusiaceae, foram isoladas cinco xantonas, 1,6-diidroxi-3,5-dimetoxixantona, 2-hidroxi-1-metoxixantona, 2,3-metilenodioxixantona, 4-hidroxi-2,3-metilenodioxixantona e 3-hidroxi-2,4-dimetoxixantona e dois triterpenos, $\alpha$-amirina e $\beta$-amirina. $\mathrm{O}$ extrato exibiu toxicidade para Artemia salina e atividade antibacteriana para Micrococcus luteus, Bacillus subitilis, Staphylococcus aureus e Streptococcus mutans, não sendo ativo para Escherichia coli, Pseudomonas aeruginosa e Salmonela choleraesuis.
\end{abstract}

Unitermos: Kielmeyera cuspidata, Clusiaceae, xantonas, triterpenos, atividade antibacteriana.

\begin{abstract}
Xanthones, triterpenes and antibacterial activity of dichloromethane extract of Kielmeyera cuspidata Saddi, Clusiaceae". From the dichloromethane extract of trunk of Kielmeyera cuspidata five xanthones were isolated, 1,6-dihydroxy-3,5-dimethoxyxanthone, 2-hydroxy1-methoxyxanthone, 2,3-methylenedioxyxanthone, 4-hydroxy-2,3-methylenedioxyxanthone and 3-hydroxy-2,4-dimethoxyxanthone and two triterpenes $\alpha$-amirin and $\beta$-amirin. The extract displayed toxicity against Artemia salina and exhibited antibacterial activity against Micrococcus luteus, Bacillus subitilis, Staphylococcus aureus and Streptococcus mutans and it was not active against Escherichia coli, Pseudomonas aeruginosa and Salmonela choleraesuis.
\end{abstract}

Keywords: Kielmeyera cuspidata, Clusiaceae, xanthones, triterpenes, antibacterial activity.

\section{INTRODUÇ̃̃OO}

Afamília de Clusiaceae engloba aproximadamente 1000 espécies em trinta gêneros distribuídos na zona pantropical. No Brasil existem dezoito gêneros e aproximadamente 150 espécies (Souza \& Lorenzi, 2005), sendo o gênero Kielmeyera endêmico da América do Sul (Bennett \& Lee, 1989). Os estudos com espécies deste gênero demonstraram uma maior freqüência de algumas classes de substâncias químicas, dentre elas estão as 4-alquil e 4-fenilcumarinas (neoflavonóides) e as xantonas, sendo as xantonas o metabólito secundário mais largamente encontrado no mesmo (Cruz et al., 2001; Moreira et al., 2002). Substâncias isoladas de espécies do gênero foram submetidas a ensaios biológicos e algumas destas apresentaram atividades antimicrobiana (Cortez et al., 1998), antioxidante (Iinuma et al., 1996), antitumoral (DallÁcqua et al., 2002), dentre outras. Os trabalhos precedentes com algumas espécies deste gênero, encontradas no cerrado e no Planalto Central Brasileiro, descrevem as xantonas como seus constituintes principais (Nagem \& Silva, 1988). Em contrapartida, nas espécies
$K$. argentea e $K$. reticulata coletadas em um habitat de restinga na costa de Bahia, Brasil, foram isoladas predominantemente 4-fenilcoumarinas (neoflavonóides) e 4- $n$-propilcumarinas (Cruz et al., 1998a e 1998b; Moreira et al., 2002).

Apesar de existirem vários relatos sobre o estudo químico com espécies do gênero (Nagem \& Silva, 1988; Gramacho et al., 1999; Cruz et al., 2001) até o presente momento este é o primeiro trabalho de avaliação química e antibacteriana realizado com a espécie K. cuspidata.

\section{MATERIAL E MÉTODOS}

\section{Equipamentos}

Os espectros de RMN foram obtidos em espectrômetro Varian - Gemini 2000 operando a 300 $\mathrm{MHz}$ para hidrogênio e a $75 \mathrm{MHz}$ para carbono-13 e as amostras foram solubilizadas em $\mathrm{CDCl}_{3}, \mathrm{CDCl}_{3}+\mathrm{DMSO}-$ $\mathrm{d}_{6}$, DMSO-d $\mathrm{d}_{6}$ e acetona-d $\mathrm{d}_{6}$.

\section{Material vegetal}


A espécie $K$. cuspidata, foi coletada em maio de 2003 em uma região de campo rupestre da Chapada Diamantina, Andaraí, Bahia, Brasil. A espécie foi identificada e catalogada pela professora Maria Lenise Silva Guedes sob o registro ALCB-061902 e está depositada no do Herbário Alexandre Leal Costa, Instituto de Biologia, Universidade Federal da Bahia, Salvador, Brasil.

\section{Extração e isolamento dos constituintes químicos}

O caule triturado da planta $(3,87 \mathrm{~kg})$ foi submetido a extrações sucesssivas com solventes orgânicos, primeiramente em hexano e depois em metanol. Foram obtidos assim, os extratos do caule em hexano (46,51 g) e o extrato em metanol $(30,14 \mathrm{~g})$. O extrato em metanol $(30,14 \mathrm{~g})$ foi submetido à partição dissolvendo o extrato em uma solução de metanol/água (8:2). A solução obtida foi submetida a extrações sucessivas com diclorometano. A fase obtida foi concentrada em evaporador rotatório obtendo-se o extrato diclorometânico (8,70 g). O extrato diclorometânico foi submetido a uma filtração em coluna de gel de sílica, usando como eluentes hexano e acetato de etila em proporções crescentes. Foram obtidas 22 frações. Essas frações foram analisadas por cromatografia em camada delgada em gel de sílica e agrupadas por similaridade em quinze frações. Algumas delas, depois de serem analisadas por $\mathrm{RMN}$ de ${ }^{1} \mathrm{H}$, foram recromatografadas em colunas sucessivas de gel de sílica eluídas com hexano/ acetato de etila em gradientes crescentes de polaridade. Da fração 4 foi obtida uma mistura de $\alpha$-amirina e $\beta$-amirina $(33,9 \mathrm{mg})$, da fração 8 foram obtidas após purificação por Cromatografia em Camada Delgada Preparativa (CCDP) em sílica gel $\mathrm{PF}_{254+366}$ eluída com hexano/diclorometano $8: 2$ as substâncias 2-hidroxi-1-metoxixantona (1,8 mg), $\beta$-amirina (5,8 mg), 1,6-diidroxi-3,5-dimetoxixantona (17,3 mg), da fração 9 após purificação por CCDP em sílica gel $\mathrm{PF}_{254+366}$ eluída com éter de petróleo/diclorometano 8:2 foi obtida a substância 1,6-diidroxi-3,5-dimetoxixantona (20,2 mg), da fração 10 foi obtida a substância 4-hidroxi2,3-metilenodioxixantona (14,5 mg) e da fração 12 foi obtida a substância 3-hidroxi-2,4-dimetoxixantona (12,2 $\mathrm{mg})$.

1,6-diidroxi-3,5-dimetoxixantona (1): $\mathrm{RMN}{ }^{1} \mathrm{H}\left(\mathrm{CDCl}_{3}\right), \delta$ (ppm): 6,47 (H-2, d, J 2,1 Hz), 6,35 (H-4, d, J 2,1 Hz), 6,98 $(\mathrm{H}-7, d, J 8,6 \mathrm{~Hz}), 7,92(\mathrm{H}-8, d, J 8,6 \mathrm{~Hz}), 12,95$ (OH-1, s), 4,10 (OMe-3,s), 3,89 (OMe-5, s). RMN C ${ }^{13}\left(\mathrm{CDCl}_{3}\right)$, $\delta$ (ppm): 163,6 (C-1), 97,2 (C-2), 166,4 (C-3), 93,0 (C-4), 157,2 (C-4a), 149,7 (C-4b), 133,7 (C-5), 154,5 (C-6), 115,0 (C-7), 121,9 (C-8), 112,5 (C-8a), 103,3 (C-8b), 180,1 (C-9), 55,8 (3-OMe), 61,9 (5-OMe) (Wolfender et al., 1991).

2-hidroxi-1-metoxixantona (2): $\mathrm{RMN}{ }^{1} \mathrm{H}$ (acetona-d $\left.\mathrm{d}_{6}\right), \delta$ (ppm): 7,35 (H-3, m), 7,25 (H-4, d, J 8,8 Hz), 7,45 (H-5, $m), 7,71$ (H-6, $d, J 6,8 \mathrm{~Hz}), 7,39(m), 8,32$ (H-8, d, J6,8 Hz),
3,89 (OH-2, s), 4,06 (1-OMe, s) (Cortez et al., 1998).

2,3-metilenodioxixantona (3): $\mathrm{RMN}{ }^{1} \mathrm{H}\left(\mathrm{CDCl}_{3}\right), \delta(\mathrm{ppm})$ : 7,67 (H-1, s), 6,92 (H-4, s), 7,47 (H-5, dl, J 8,8 Hz), 7,71 (H-6, $m), 7,38$ (H-7, tl, J 8,8 Hz), 8,33 (H-8, dd, J 8, 8 e 1,8 $\mathrm{Hz}), 6,12\left(\mathrm{CH}_{2}-10, s\right)$ (Cruz et al., 2001).

4-hidroxi-2,3-metilenodioxixantona (4): $\mathrm{RMN}^{1} \mathrm{H}$ (DMSO$\left.\mathrm{d}_{6}\right), \delta$ (ppm): 7,01 (H-1, s), 7,62 (H-5, dl, J 8,8 Hz), 7,42 (H-6, tl, J 8,8 Hz), 7,79 (H-7, t, 8,8 e 2,1 Hz), 8, 10 (H-8, $d l, J 8,8 \mathrm{~Hz}), 6,10\left(\mathrm{CH}_{2}-10, s\right)$. RMN C ${ }^{13}\left(\mathrm{DMSO}_{-} \mathrm{d}_{6}\right), \delta$ (ppm): 93,8 (C-1), 145,0 (C-2), 130,4 (C-3), 141,0 (C-4), 146,1 (C-4a), 155,2 (C-4b), 118,7 (C-5), 135,4 (C-6), 124,9 C-7), 126,5 (C-8), 121,1 (C-8a), 116,6 (C-8b), 174,9 (C-9), 103,2 (C-10) (Gottlieb et al., 1971).

3-hidroxi-2,4-dimetoxixantona (5): $\mathrm{RMN}{ }^{1} \mathrm{H}\left(\mathrm{CDCl}_{3}+\right.$ DMSO-d $\left.{ }_{6}\right), \delta$ (ppm): 7,47 (H-1, s), 7,53 (H-5, dd, J 8,8 e 1,6 Hz), 7,67 (H-6, dt, J 8,8; 6,5 e 1,6 Hz), 7,34 (H-7, $t l$, J 8,8 Hz), 8,29 (H-8, dd, J 8,8 e 1,6 Hz), 6,03 (OH-3, $s), 3,97$ (2-OMe, $s), 4,05$ (4-OMe, s). RMN C ${ }^{13}\left(\mathrm{CDCl}_{3}\right.$ $\left.+\mathrm{DMSO}_{6}\right), \delta(\mathrm{ppm}): 100,4$ (C-1), 145,5 (C-2), 145,9 (C-3), 134,8 (C-4), 146,2 (C-4a), 155,6 (C-4b), 117,7 (C-5), 133,7 (C-6), 123,5 C-7), 126,1 (C-8), 121,0 (C-8a), 113,6 (C-8b), 175,7 (C-9), 56,1 (2-OMe), 61,2 (4-OMe) (Gottlieb et al., 1971).

\section{Bioensaios}

Microdiluição em caldo - Determinação da Concentração Inibitória Minima (CIM)

O teste de microdiluição em caldo para a determinação da CIM, foi realizado segundo a metodologia de Bicalho et al. (2003). Inicialmente, foram distribuídos $100 \mu \mathrm{L}$ de caldo nutriente (Oxoid) em placas de 96 poços. Na primeira fileira de poços, foram adicionados, em triplicata, $100 \mu \mathrm{L}$ da solução estoque da fase em

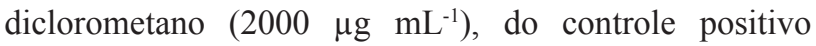
(cloranfenicol, $2000 \mu \mathrm{g} \mathrm{mL} \mathrm{m}^{-1}$ ) e do controle do diluente (água:dimetilsulfóxido: 20\%, v/v). Em seguida, diluições seriadas, com fator de diluição de dois, foram realizadas e, finalmente, foram adicionados $100 \mu \mathrm{L}$ da suspensão da bactéria $\left(10^{6}\right.$ células $\left.\mathrm{mL}^{-1}\right)$. A amostra e o controle positivo foram testados em concentrações variando de $500 \mu \mathrm{g} \mathrm{mL}^{-1}$ a $3,9 \mu \mathrm{g} \mathrm{mL} \mathrm{mL}^{-1}$. A leitura da concentração inibitória mínima (CIM) foi efetuada visualmente após incubação das bactérias durante $24 \mathrm{~h}$, a $37^{\circ} \mathrm{C}$. As bactérias utilizadas nos experimentos foram Bacillus subtilis ATCC 6633, Micrococus luteus ATCC 10240, Staphylococcus aureus ATCC 6538, Streptococcus mutans ATCC 25175, Escherichia coli ATCC 94863, Salmonela choleraesuis ATCC 14028 e Pseudomonas aeruginosa.

\section{Letalidade contra Artemia salina}

No bioensaio de letalidade para larvas de Artemia salina foi utilizada a metodologia descrita na literatura (Meyer et al., 1982). Os extratos foram dissolvidas em 
dimetilsulfóxido e diluídos em solução salina $(\mathrm{NaCl})$. Larvas de A. salina (10 por frasco) foram colocadas em contato com os extratos nas concentrações finais 10 , 100 e $1000 \mu \mathrm{g} / \mathrm{mL}$, durante $24 \mathrm{~h}$. Após este período, os sobreviventes foram contados e a $\mathrm{CL}_{50}$ foi obtida pelo programa PROBIT.

\section{RESULTADOS E DISCUSSÃO}<smiles>COc1cc(O)c2c(=O)c3ccc(O)c(OC)c3oc2c1</smiles><smiles>O=c1c2ccccc2oc2c(O)c3c(cc12)OCO3</smiles><smiles>COc1cc2c(=O)c3ccccc3oc2c(OC)c1O</smiles>

5
O fracionamento por cromatografia em coluna de gel de sílica do extrato em diclorometano levou ao isolamento e identificação de cinco xantonas 1,6-diidroxi3,5-dimetoxixantona (1), 2-hidroxi-1-metoxixantona (2), 2,3-metilenodioxixantona (3), 4-hidroxi-2,3metilenodioxixantona (4) e 3-hidroxi-2,4-dimetoxixantona (5), além dos triterpenos $\alpha$-amirina e $\beta$-amirina. Estas substâncias tiveram suas estruturas determinadas através da análise de seus espectros de RMN e por comparação<smiles>COc1c(O)ccc2oc3ccccc3c(=O)c12</smiles>

2<smiles>C[C@H]1CCC2(C)CC[C@H]3C(=CCC4[C@@]5(C)CC[C@H](O)C(C)(C)[C@H]5CC[C@]43C)C2[C@@H]1C</smiles>

$\alpha$-amirina<smiles>O=c1c2ccccc2oc2cc3c(cc12)OCO3</smiles>

3

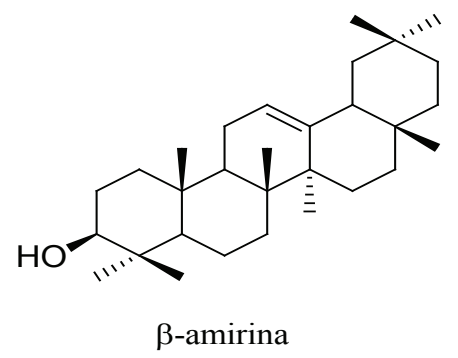

de seus dados de RMN com dos dados publicados na literatura.

No bioensaio para a determinação da CIM, o extrato diclorometânico foi testado contra as bactérias B. subtilis, S. aureus, S. mutans, M. luteus, E. coli, P. aeruginosa, S. choleraesuis. Os resultados (Tabela 1) demonstraram que o extrato foi ativo apenas contra as bactérias Gram positivas (B. subtilis, S. aureus, S. mutans, M. luteus) e não inibiu o crescimento das bactérias Gram negativas (E. coli, P. aeruginosa, S. choleraesuis) em concentrações menores do que $500 \mu \mathrm{g} . \mathrm{mL}^{-1}$. O extrato foi mais ativo contra $\mathrm{M}$. luteus exibindo uma CIM de 7,8 $\mu \mathrm{g} . \mathrm{mL}^{-1}$ frente a $3,9 \mu \mathrm{g} \cdot \mathrm{mL}^{-1}$ do cloranfenicol, antibiótico utilizado como controle positivo. Este extrato foi altamente

Tabela 1. Concentração Inibitória Mínima (MIC) do extrato diclorometânico de K. cuspidata.

\begin{tabular}{lcc}
\hline \multicolumn{1}{c}{ Microorganismo } & Extrato diclorometânico $\left(\mu \mathrm{g} \cdot \mathrm{mL}^{-1}\right)$ & Cloranfenicol $\left(\mu \mathrm{gg} \cdot \mathrm{mL}^{-1}\right)$ \\
\hline Bacillus subtilis ATCC 6633 & 15,6 & 3,9 \\
Staphylococcus aureus ATCC 6538 & 31,2 & 7,8 \\
Streptococcus mutans ATCC 5175 & 31,2 & 3,9 \\
Micrococcus luteus ATCC 10240 & 7,8 & 3,9 \\
Escherichia coli ATCC 94863 & $>500$ & 3,9 \\
Pseudomonas aeruginosa & $>500$ & 62,2 \\
Salmonela choleraesuis ATCC 14028 & $>500$ & 3,9 \\
\hline
\end{tabular}

Os experimentos foram feitos em duplicata.

$>500 \mu \mathrm{g} \cdot \mathrm{mL}^{-1}$ : não ocorreu inibição em concentração menor que $500 \mu \mathrm{g} \cdot \mathrm{mL}^{-1}$. 
tóxico para Artemia salina, apresentando um $\mathrm{LC}_{50}$ of 19,9 $\mathrm{mg} \mathrm{mL} \mathrm{m}^{-1}$.

\section{AGRADECIMENTOS}

Os autores agradecem a FAPESB, CNPq e FINEP pelo suporte financeiro e bolsas de estudo.

\section{REFERÊNCIAS}

Bennett GJ, Lee HH 1989. Xanthones from Guttiferae. Phytochemistry 28: 967-998.

Bicalho B, Gonçalves RAC, Zibordi AP, Manfio GP, Marsaioli A 2003. Antimicrobial compounds of fungi vectored by Clusia ssp. (Clusiaceae) pollinating bees. Verlag $Z$ Naturforsch 58c: 746-751.

Cortez DAG, Young MCM, Marston A, Wolfender JL, Hostettmann K 1998. Xanthones, triterpenes and a biphenyl from Kielmeyera coriacea. Phytochemistry 47: 1367-1374.

Cruz FG, David JM, Guedes MLS, Chávez JP, Santos NAS 1998a. Coumarins from Kielmeyera argentea. Phytochemistry 48: 703-706.

Cruz FG, David JM, Guedes MLS, Chávez JP, Moreira LM 1998b. Coumarins from Kielmeyera reticulata. Phytochemistry 47: 1363-1366.

Cruz FG, Silva-Neto JT, Guedes MLS 2001. Xanthones and coumarins de Kielmeyera lathrophyton. J Braz Chem Soc 12: $117-122$.

Dall'Ácqua S, Innocenti G, Viola G, Piovan A, Caniato R, Cappelletti EM 2002. Cytotoxic compounds from Polygala vulgaris. Chem Pharm Bull 50: 1499-1501.

Gottlieb OR, Mesquita AAL, Nagem TJ 1971. Xanthones from Kielmeyera rubriflora. Phytochemistry 10: 2253-2255.

Gramacho RS, Nagem TJ, Oliveira TT, Queiroz MELR, Neves AA, Saddi N 1999. Phenylcoumarins from Kielmeyera elata. Phytochemistry 51: 579-581.

Iinuma M, Tosa H, Tanaka T, Kanamaru S, Asai F, Kobayashi Y, Miyauchi K, Shimano R 1996. Antibacterial activity of some Garcinia benzophenone derivatives against methicillin-resistant Staphylococcus aureus. Biol Pharm Bull 19: 311-314.

Meyer BN, Ferrigni NR, Putnam JE, Jacobsen LB, Nichols DE, McLaughlin JL 1982. Brine shrimp - a convenient general bioassay for active-plant constituents. Planta Med 45: 31-34.

Moreira LM, Cruz FG, Guedes MLS, Santos NS 2002. Additional coumarins from Kielmeyera reticulata. J Braz Chem Soc 13: 704-707.

Nagem TJ, Silva MA 1988. Xanthones and phenylcoumarins from Kilmeyera pumila. Phytochemistry 27: 2961-2962.

Souza V, Lorenzi H 2005. Botânica sistemática: guia ilustrado para identificação de famílias de angiospermas da flora brasileira. São Paulo: Instituto Plantarum, p. 348.

Wolfender JL, Hamburger M, Msonth JD, Hostettmann K 1991. Xanthones from Chironia krebsii. Phytochemistry 30: 3625-3629. 